\title{
MAXIMAL QUOTIENTS OF SEMIPRIME PI-ALGEBRAS
}

BY

\section{LOUIS HALLE ROWEN(1)}

ABSTRACT. J. Fisher [3] initiated the study of maximal quotient rings of semiprime PI-rings by noting that the singular ideal of any semiprime PI-ring $R$ is 0 ; hence there is a von Neumann regular maximal quotient ring $Q(R)$ of $R$. In this paper we characterize $Q(R)$ in terms of essential ideals of $C=$ cent $R$. This permits immediate reduction of many facets of $Q(R)$ to the commutative case, yielding some new results and some rapid proofs of known results. Direct product decompositions of $Q(R)$ are given, and $Q(R)$ turns out to have an involution when $R$ has an involution.

1. Introduction. Let $\Omega$ be a commutative algebra with 1 and let $R$ be a semiprime $\Omega$-algebra, not necessarily with 1 (by semiprime we mean $R$ has no nonzero nilpotent ideals, where all ideals are understood to be $\Omega$-invariant; equivalently, the intersection of the prime ideals of $R$ is 0 ). Let the standard polynomial on $k$ letters $s_{k}\left(X_{1}, \cdots, X_{k}\right) \equiv \Sigma_{\pi}(s g \pi) X_{\pi 1} \cdots X_{\pi k}, \pi$ ranging over the permutations of $(1, \cdots, k)$; a polynomial $f\left(X_{1}, \ldots, X_{m}\right)$ (with coefficients in $\Omega$ ) is an identity of $R$ if, evaluated in $R, f\left(X_{1}, \cdots, X_{m}\right)=0$, each $r_{1}, \cdots, r_{m}$ in $R$. The semiprime algebra $R$ is a PI -algebra of degree $n$ if $S_{2 n}$ is an identity of $R$ but $S_{2 n-2}$ is not an identity of $R$. Throughout this paper we assume $R$ is a semiprime PI-algebra of finite degree $n$, and we let $C=$ cent $R$. Formanek [4] has shown there exists a polynomial $g\left(X_{1}, \ldots, X_{n+1}\right)$ with integral coefficients, one of which is \pm 1 , such that each of $X_{2}, \cdots, X_{n+1}$ has degree 1 in each monomial of $g$; moreover, evaluated in $R, g\left(r_{1}, \cdots, r_{n+1}\right) \in C$ for each $r_{1}, \cdots, r_{n+1}$ in $R$, and there exist $r_{1}, \cdots, r_{n+1}$ in $R$ such that $g\left(r_{1}, \cdots, r_{n+1}\right) \neq 0$ (in particular $C \neq 0)$. An application of Formanek's polynomials is

Theorem $A$ (Rowen [8]). If $A$ is a nonzero ideal of $R$ then $A \cap C \neq 0$.

Now for subsets $V, W$ of $R$, define $A \mathrm{nn}_{V} W=\{v \in V \mid W v=0\}$ and $\operatorname{Ann}_{V}^{\prime} W=$

Received by the editors August 7, 1973.

AMS (MOS) subject classifications (1970). Primary 16A08, 16A12, 16A38; Secondary 16A28, 16A48.

Key words and phrases. Essential, identity, injective hull, involution, maximal quotient algebra, PI-algebra, semiprime, singular ideal.

(1) The bulk of the work on this paper was done while the author was a doctoral candidate at Yale University under the guidance of $\mathrm{N}$. Jacobson, and was supported in part by NSF Grant GP-33591. 
$\{v \in V \mid v W=0\}$. If $V=R$ then the subscript will be omitted. Clearly, for any ideal $A$ of $R$, Ann $A=A_{n n}^{\prime} A$ (Proof. $\left(A A^{\prime} n^{\prime} A\right)^{2}=A\left(\left(A_{n n}^{\prime} A\right) A\right) A n n^{\prime} A=0$, so $A \mathrm{Ann}^{\prime} A=0$ since $R$ is semiprime. Hence $A \mathrm{nn}^{\prime} A \subseteq A \mathrm{nn} A$ and, symmetrically, Ann $A \subseteq A n n^{\prime} A$.) Similarly, one sees that for any ideals $A, B$ of $R, A B=0 \Leftrightarrow$ $A \cap B=0$.

Call a (left, right, 2-sided) ideal $J$ of $R$ (left, right, 2-sided) essential if $J \cap B \neq 0$ for all nonzero (left, right, 2-sided) ideal of $R$. (The word "2-sided" will often be omitted for convenience.) An ideal $J$ of $R$ is essential if and only if $A \mathrm{nn} J=0$, if and only if $A n n^{\prime} J=0$ (by the preceding paragraph); we conclude each essential ideal of $R$ is left essential and right essential. (Indeed, suppose $J$ is an essential ideal of $R$ and $B$ is a left ideal of $R$ such that $J \cap B=0$. Then $J B \subseteq J \cap B=0$, so $B \subseteq A n n J=0$.) By a left essential ideal we mean a left essential left ideal. If $J$ is a left essential ideal of $R$ then $A n n^{\prime} j=0$. Let $Z=\{r \in R \mid$ there exists a left essential ideal $J$ of $R$ such that $r \in A n n J\}$. $Z$ is well known to be an ideal of $R$, called the left singular ideal. The right singular ideal $Z^{\prime}$ is defined analogously.

Proposition 1 (Fisher [3]). $Z=Z^{\prime}=0$.

Proof (Martindale [6]). Let $c \in Z \cap C$. Then $c \in \mathrm{Ann}^{\prime} J$ for some left essential ideal of $R$. But $A n n^{\prime} J=0$, so $Z \cap C=0$. Therefore, $Z=0$ by Theorem $\Lambda$. Likewise $Z^{\prime}=0$. Q.E.D.

In this case, it is well known (cf. Johnson [S]) that the left injective hull of $R$ has a natural ring structure $Q(R) . Q(R)$ can be characterized in terms of essential ideals, as follows (cf. Martindale [6]):

(a) There is a canonical injection $R \hookrightarrow Q(R)$ by which we view $R \subseteq Q(R)$.

(b) For any left essential ideal $J$ of $R$ and for any $f$ in $\operatorname{Hom}_{R}(J, R)$ (as left $R$-modules), there exists $q$ in $Q(R)$ such that $x q=f(x)$, all $x$ in $J$.

(c) For any given $q$ in $Q(R)$ there is a left essential ideal $J$ of $R$ such that $J q \subseteq R$.

(d) $q=0$ if and only if $J q=0$ for some left essential ideal $J$ of $R$.

There is a natural way to extend the algebra structure of $R$ to $Q(R)$. Namely, given $q$ in $Q(R), \omega$ in $\Omega$, let $J$ be a left essential ideal of $R$ such that $J q \subseteq R$, and define $f$ in $\operatorname{Hom}_{R}(J, R)$ by $f(x)=\omega(x q)$, all $x$ in $J$. By (b), we may pick $q_{1}$ in $Q(R)$ such that $x q_{1}=f(x)$, all $x$ in J; define $\omega q$ to be $q_{1}$. To see that $\omega q$ is well defined, suppose $J^{\prime}$ is another left essential ideal of $R$ such that $J^{\prime} q \subseteq R$, and define $f^{\prime}$ in $\operatorname{Hom}_{R}\left(J^{\prime}, R\right)$ by $f^{\prime}(x)=\omega(x q)$, all $x$ in $J^{\prime}$; let $q_{1}^{\prime}$ in $Q$ be such that $x q_{1}^{\prime}=f^{\prime}(x)$, all $x$ in $J^{\prime}$. For all $x$ in $J \cap J^{\prime}, x q_{1}=f(x)=\omega(x q)=f^{\prime}(x)=x q_{1}^{\prime}$, so $\left(J \cap J^{\prime}\right)\left(q_{1}-q_{1}^{\prime}\right)=0$. Since $J \cap J^{\prime}$ is left essential, $q_{1}=q_{1}^{\prime}$ by (d); hence $\omega q$ is well defined, extending the algebra structure on $R$. Similar verifications show that $Q(R)$ is now an algebra, called henceforth the maximal left quotient 
algebra of $R$; for any $f \in \operatorname{Hom}(J, R), J$ left essential, we have $f(\omega x)=\omega f(x)$ for all $\omega$ in $\Omega, x$ in $J$.

2. A central characterization of $Q(R)$.

Lemma 1 (Martindale [6]). Any left essential ideal $J$ of $R$ is itself a semiprime PI-algebra, and cent $J=J \cap C$.

Proof. Straightforward application of Proposition 1. The following lemma is also known by Martindale [6], but a different proof is used to avoid reliance on the other results in [6].

Lemma 2.(i) If $J$ is a left essential ideal of $R$ then $J \cap C$ intersects nontrivially all ideals of $R$. Hence $(J \cap C) R$ is 2-sided essential.

(ii) A left ideal $J$ of $R$ is left essential if and only if $(J \cap C)$ is essential in $C$.

Proof. (i) Suppose $B$ is an ideal of $R$ such that $(J \cap C) \cap B=0$. Then $(J \cap C) \cap(J \cap B)=0$, so by Theorem $A$ applied to the semiprime PI-algebra $J$ (with center $J \cap C$ ), we conclude $J \cap B=0$. Hence $B=0$, so (i) follows immediately.

(ii) Suppose $J$ is left essential and let $B=\Lambda \mathrm{nn}_{C}(J \cap C)$. Clearly $B R(J \cap C)$ $=0$, so $(B R \cap(J \cap C))^{2}=0$; hence $B R \cap(J \cap C)=0$, implying $B R=0$ from (i). Therefore $B=0$, so $J \cap C$ is essential in $C$.

Conversely, suppose $(J \cap C)$ is essential in $C$ and let $B$ be a left ideal of $R$ such that $J \cap B=0$. Then $(B R \cap C)(J \cap C) \subseteq B(J \cap C) R \subseteq(B \cap J) R=0$, so $B R \cap$ $C=0$. Therefore $B R=0$, by Theorem $A$, so $B=0$ and $J$ is left essential. Q.E.D.

The routine preliminaries have been set for the main theorem:

Theorem 1. $Q(R)$ is characterized by the following properties:

(i) There is a canonical injection $R \hookrightarrow Q(R)$ sending $C$ into cent $Q(R)$.

(ii) For any essential ideal $E$ of $C$ and for any $f$ in $\operatorname{Hom}_{C}(E, R)$, one can find $q$ in $Q(R)$ such that $x q=f(x)$, all $x$ in $E$.

(iii) For any $q$ in $Q(R), E q \subseteq R$ for some essential ideal $E$ of $C$.

(iv) $q=0$ if and only if $E q=0$ for some essential ideal $E$ of $C$.

Proof. First we show (a)-(d) of the previous characterization imply (i)-(iv).

(i) $R \subseteq Q(R)$; we claim $C \subseteq$ cent $Q(R)$. Choose $c$ in $C, q$ in $Q(R)$. By (c), $J q \subseteq R$ for some left essential ideal $J$ of $R$, so, for all $x$ in $J, 0=(x q) c-c(x q)=$ $x q c-(c x) q=x q c=x(q c-c q)$, implying $q c-c q=0$ by (d). Hence $C \subseteq$ cent $Q(R)$.

(ii) Let $E$ be an essential ideal of $C$. Then $C \cap R E$ is surely essential in $C$, so, by Lemma 2, RE is essential in $R$. Given $f$ in $\operatorname{Hom}_{C}(E, R)$ we wish to define $f^{\prime}: R E \rightarrow R$ by $f^{\prime}\left(\Sigma r_{i} c_{i}\right)=\Sigma r_{i} f\left(c_{i}\right)$, all $c_{i}$ in $E$, all $r_{i}$ in $R$. To check 
that $f^{\prime}$ is well defined, let $B=\left\{\Sigma r_{i} f\left(c_{i}\right) \mid \Sigma r_{i} c_{i}=0, r_{i}\right.$ in $R, c_{i}$ in $\left.E\right\}$, an ideal of $R$. If $B \neq 0$ then $B \cap C \neq 0$ by Theorem $A$, so $B \cap C \cap E \neq 0$ and we could choose nonzero $b=\Sigma r_{i} f\left(c_{i}\right)$ in $B \cap \cap \cap C$, with $\Sigma r_{i} c_{i}=0$. But then $b^{2}=$ $b \Sigma r_{i} f\left(c_{i}\right)=\Sigma r_{i} f\left(b c_{i}\right)=\Sigma r_{i} f\left(c_{i} b\right)=\left(\Sigma r_{i} c_{i}\right) f(b)=0$, contrary to $C$ being semiprime. Hence $B=0$ and $f^{\prime}$ is a well-defined element of $\operatorname{Hom}_{R}(R E, R)$. By (b), there exists $q$ in $Q(R)$ such that $x q=f^{\prime}(x)$ for all $x$ in $R E$, implying (by (d)) $x q=f^{\prime}(x)$ for all $x$ in $E$.

(iii) By (c), $J q \subseteq R$ for some left essential ideal $J$ of $R$. Let $E=J \cap C$, an essential ideal in $C$ by Lemma 2.

(iv) Immediate from (d) and Lemma 2.

Thus, the left maximal quotient algebra $Q(R)$ satisfies (i)-(iv). Conversely, assume some algebra $Q$ satisfies (i)-(iv). We shall show $Q=Q(R)$ by verifying (a)-(d).

(a) Immediate from (i).

(b) Suppose $J$ is left essential in $R$ and $f \in \operatorname{Hom}_{R}(J, R)$. Then, by Lemma 2, $E=J \cap C$ is essential in $C$ and surely $f \in \operatorname{Hom}_{C}(E, R)$. By (ii), there exists $q$ in $Q$ such that $f(c)=c q$, all $c$ in $E$. For all $x$ in $J$, all $c$ in $E, c(f(x)-x q)=$ $f(c x)-c x q=f(x c)-x c q=x(f(c)-c q)=0$, so $E(f(x)-x q)=0$, all $x$ in $J$. Hence by (iv), $f(x)=x q$, all $x$ in $J$.

(c) Immediate from (iii) and Lemma 2.

(d) Immediate from (iv) and Lemma 2. Q.E.D.

Corollary 1 (Martindale [6, Theorem 5]), $Q(R)$ is also the right maximal quotient algebra of $R$.

Proof. Conditions (i)-(iv) are left-right symmetric. Q.E.D.

Martindale also has shown $Q(R)$ satisfies all multilinear identities of $R$. Call a polynomial $f\left(X_{1}, \cdots, X_{m}\right)$ bomogeneous if each monomial of $f$ has the same total degree.

Corollary 2. Eacb homogeneous identity of $R$ is an identity of $Q(R)$.

Proof. Let $f\left(X_{1}, \cdots, X_{m}\right)$ be a homogeneous identity of $R$, of total degree $d$. We wish to show, given any $q_{1}, \cdots, q_{m}$ in $Q(R)$, that $f\left(q_{1}, \cdots, q_{m}\right)=0$. By Theorem 1 (iii) there are essential ideals $E_{i}$ of $C$ such that $E_{i} q_{i} \subseteq R, 1 \leq i \leq m$. Let $E=E_{1} \cap \ldots \cap E_{m}$, an essential ideal of $C$. For each $c$ in $E, 0=$ $f\left(c q_{1}, \cdots, c q_{m}\right)=c^{d} f\left(q_{1}, \cdots, q_{m}\right)$, so $0=\hat{E} f\left(q_{1}, \cdots, q_{m}\right)$, where $\hat{E}$ is the ideal of $C$ generated by $\left\{c^{d} \mid c \in E\right\}$. But $\hat{E}$ is essential in $C$; indeed, for any nonzero ideal $B$ of $C$ we can pick $b \neq 0$ in $B \cap E$, and then $0 \neq b^{d} \in B \cap \hat{E}$. Hence $f\left(q_{1}, \cdots, q_{m}\right)=0$ by Theorem 1 (iv). Q.E.D.

Corollary 3 (Armendariz-Steinberg [2]). cent $Q(R)=Q(C)$. 
Proof. Let $C^{\prime}=$ cent $Q(R)$. We need to verify (i)'-(iv) ${ }^{\prime}$, obtained from conditions (i)-(iv) of Theorem 1 by replacing $R$ by $C$.

(i) $C \hookrightarrow C^{\prime}$ is part of (i).

(ii) Given $E$ essential in $C$ and $f$ in $\operatorname{Hom}_{C}(E, C)$, Theorem 1 (ii) provides $q$ in $Q(R)$ such that $x q=f(x)$, all $x$ in $E$. It suffices to show $q \in C^{\prime}$. Note $E q \subseteq$ $C \subseteq C^{\prime}$; for all $c$ in $E$, all $q^{\prime}$ in $Q(R), 0=(c q) q^{\prime}-q^{\prime}(c q)=c\left(q q^{\prime}-q^{\prime} q\right)$, so $q q^{\prime}-q^{\prime} q=0$ (by (iv)), all $q^{\prime}$ in $Q(R)$, implying $q \in C^{\prime}$.

(iii) ${ }^{\prime}$, (iv)' are immediate respectively from (iii), (iv). Q.E.D.

Incidentally, if $R$ is the infinite direct sum $\bigoplus M_{n}(Q)$ then $Q(R)$ is the infinite direct product $\Pi M_{n}(Q)$, so $Q(C) R \neq Q(R)$ in this case (example due, I believe, to R. Snider).

Proposition 2. For $q$ in $Q(R), q \in$ cent $Q(R)$ if and only if there is a left essential ideal $J$ of $R$ such that $q x-x q=0$, all $x$ in $J$.

Proof. $\Leftrightarrow$ ) Obvious.

$\Leftrightarrow$ Pick $q^{\prime}$ arbitrarily from $Q(R)$ and let $E$ be an essential ideal of $C$ such that $E q^{\prime} \subseteq R$ (cf. Theorem 1 (iii)). Then $E^{\prime}=(J \cap C) E$ is essential in $C$ and, for all $c$ in $E^{\prime}$, we have $c q^{\prime} \in J$ and $c\left(q q^{\prime}-q^{\prime} q\right)=q\left(c q^{\prime}\right)-\left(c q^{\prime}\right) q=0$. Hence $q q^{\prime}$ $-q^{\prime} q=0$, all $q^{\prime}$ in $Q(R)$, implying $q \in$ cent $Q(R)$. Q.E.D.

Theorem 2. Let $J$ be a 2-sided essential ideal of $R$. For any bimodule bomomorphism $f: J \rightarrow R$ there exists $q$ in cent $Q(R)$ such that $f(r)=r q$, all $r$ in $J$.

Proof. Since $f$ is a left module homomorphism and $J$ is left essential, there exists $q$ in $Q(R)$ such that $f(r)=r q$, all $r$ in $J$. For all $x, r$ in $J, x r q=x f(r)=$ $f(x r)=f(x) r=x q r$, so $x(r q-q r)=0$, implying $r q-q r=0$, all $r$ in $J$. By Proposition 2 $q \in$ cent $Q(R)$. Q.E.D.

Remark. One could parallel the proof of Theorem 1 to show $Q(R)$ is actually the $Q_{0}(R)$ of Amitsur [1]. Hence, [1, Theorem 3] implies Proposition 2 and Theorem 2. Similarly, [1, Theorem 5] yields a nice proof that $Q(R)$ is von Neumann regular, a fact observed in general (for rings with zero left singular ideal) by Johnson [5].

3. Structure of $Q(R)$. In this section we assume $1 \in R$ and give two direct sum decompositions of $Q(R)$. (Note 1 is also the multiplicative unit of $Q(R)$.) The point of departure is the easily verified

Theorem B. Viewing a left essential ideal $J$ of $R$ as a semiprime PI-algebra (by Lemma 1), we bave $Q(J) \approx Q(R)$.

Corollary 4. If $A, A^{\prime}$ are ideals of $R$ such that $A^{\prime}=A \operatorname{nn} A$ and $A=A \operatorname{nn} A^{\prime}$, then $Q(R) \approx Q(R / A) \oplus Q\left(R / A^{\prime}\right)$.

Proof. Given in [9, Theorem 4]. Like Theorem B, one does not need the 
assumption $R$ is a PI-ring but only requires that $R$ has zero left singular ideal.

Say a prime ideal $P$ of $R$ has degree $j$ if $R / P$ has degree $j$. Let $N_{n}$ be the intersection of those prime ideals of $R$ with degree $n$, and, for $j<n$, let $N_{j}=$ $\bigcap\left\{P\right.$ prime in $R$ of degree $j \mid P \nsupseteq N_{i}$, all $\left.i>j\right\}$. Clearly, $N_{1} \cap \ldots \cap N_{n}=0$.

Theorem 3. $Q(R) \approx Q\left(R / N_{1}\right) \oplus \cdots \oplus Q\left(R / N_{n}\right)$.

Proof. First we show $Q(R) \approx Q\left(R / N_{n}^{\prime}\right) \oplus Q\left(R / N_{n}\right)$, where $N_{n}^{\prime}=\bigcap_{i=1}^{n-1} N_{i^{\circ}}$ In view of Corollary 4, it suffices to show $N_{n}^{\prime}=\operatorname{Ann} N_{n}$ and $N_{n}=\Lambda$ nn $N_{n}^{0}$. Since $N_{n} N_{n}^{\prime}=0, N_{n}^{\prime} \subseteq \operatorname{Ann} N_{n}$. On the other hand, it is easy to see $N_{n}^{\prime}=\bigcap\left\{P \mid P \nsupseteq N_{n}\right\}$. For $P \nsupseteq N_{n}$, however, Ann $N_{n} \subseteq P$ since $N_{n}$ Ann $N_{n}=0 \subseteq P$; hence Ann $N_{n} \subseteq N_{n}^{n^{*}}$ Analogously, it is clear $N_{n} \subseteq$ Ann $N_{n}^{\prime}$. Since $R / N_{n}^{n}$ has degree $\leq n-1, P \nsupseteq N_{n}^{n}$ for each prime $P$ of degree $n$, so, arguing as above, we have $A_{n n} N_{n}^{\prime} \subseteq \cap\{P$ prime of degree $n\}=N_{n}$.

So $Q(R) \approx Q\left(R / N_{n}^{\prime}\right) \oplus Q\left(R / N_{n}\right)$. Since $R / N_{n}^{\prime}$ has degree $\leq n-1$, the theorem follows by induction on $n$. Q.E.D.

Armendariz-Steinberg [2] proved $Q(R)$ is a finite direct sum of Azumaya algebras of finite rank; we are now in a position to develop a straightforward proof of this fact, displaying at the same time the structure involved.

Let $g\left(X_{1}, \cdots, X_{n+1}\right)$ be the Formanek polynomial described in $\$ 1 ; g$ happens to be homogeneous of total degree $n^{2}$ (cf. [4]). Let $I_{g}(R)=\left\{g\left(r_{1}, \cdots, r_{n+1}\right) \mid\right.$ all $r_{1}, \cdots, r_{n+1}$ in $\left.R\right\}$; note that $c g\left(r_{1}, \cdots, r_{n+1}\right)=g\left(r_{1}, \cdots, r_{n+1} c\right)$ for all $c$ in $C$, so $I_{g}(R)$ is a monoid ideal of the (multiplicative) monoid $C$. Let $I_{g}^{\prime}(R)$ be the additive subgroup generated by $I_{g}(R) ; I_{g}^{\prime}(R)$ is an ideal in $C$, and the prime ideals of $R$ containing $I_{8}(R)$ are precisely those primes of degree $\leq n-1$. Also observe for any central idempotent $e, e R$ is a semiprime PI-algebra of degree $\leq n$, with multiplicative unit $e$.

Definition. $R$ is stable if $1 \in I_{\mathcal{B}}(R)$.

Lemma 3. If $e \in I_{g}(R)$ and $e$ is a nonzero idempotent then $e R$ is stable of degree $n$; i.e. $e \in I_{\varepsilon}(e R)$.

Proof. Let $e=g\left(r_{1}, \cdots, r_{n+1}\right)$. Then $g\left(e r_{1}, \ldots, e r_{n+1}\right)=e^{n^{2}} g\left(r_{1}, \cdots, r_{n+1}\right)=$ $e e=e$. Q.E.D.

Theorem 4. (i) If every nonzero ideal of $C$ contains a nonzero idempotent of $I_{g}(R)$ then $Q(R)$ is stable.

(ii) If $I_{B}^{\prime}(R)$ is essential in $C$ then $Q(R)$ is stable.

Proof. (i) Using Zom's lemma we find a collection of idempotents $e_{\lambda}$ in $I_{g}(R)$ such that $\bigoplus e_{\lambda} R$ is an essential ideal of $R$. Then $Q(R) \approx Q\left(\bigoplus_{\lambda} e_{\lambda} R\right) \approx$ $\Pi_{\lambda} Q\left(e_{\lambda} R\right)$ canonically, so $\Pi_{\lambda} e_{\lambda}=1$ in $Q(R)$. But, by Lemma 3, $e_{\lambda} \in I_{g}\left(e_{\lambda} R\right) \subseteq$ $I_{g}\left(Q\left(e_{\lambda} R\right)\right)$, so $\Pi_{\lambda} e_{\lambda} \in I_{g}\left(\Pi Q\left(e_{\lambda} R\right)\right)$. Hence $Q(R)$ is stable.

(ii) If $I_{\mathcal{g}}^{\prime}(R)$ is essential in $C$, then Theorem 1 (iii) implies $I_{g}^{\prime}(Q(R))$ is 
essential in cent $Q(R)$. Since $Q(R)=Q(Q(R))$, we may replace $R$ by $Q(R)$ and assume $C$ is von Neumann regular (in view of Corollary 3 ). We shall conclude the proof of (ii) by showing that the hypothesis of part (i) is now satisfied. Indeed, let $A$ be a nonzero ideal of $C$. Choose $a \neq 0$ in $A$ and $r_{1}, \cdots, r_{n+1}$ in $R$ such that $\operatorname{ag}\left(r_{1}, \cdots, r_{n+1}\right) \neq 0$ (possible since $0=\operatorname{Ann} I_{g}^{\prime}(R)=\operatorname{Ann} I_{g}^{(R)}\left(R\right.$ ). Let $a^{\prime}=$ $a g\left(r_{1}, \cdots, r_{n+1}\right)=g\left(r_{1}, \cdots, a r_{n+1}\right) \in A \cap I_{g}$. Since $C$ is von Neumann regular, there exists $d$ in $C$ such that $a^{\prime} d a^{\prime}=a^{\prime}$. But $a^{\prime} d$ is a nonzero idempotent of $A \cap I_{B}$, as desired. Q.E.D.

Theorems 3 and 4 combine to show $Q(R)$ is always a direct sum of the stable semiprime PI-algebras $Q\left(R_{1}\right), \cdots, Q\left(R_{n}\right)$. But every stable semiprime PI-algebra is Azumaya of finite rank, by the celebrated Artin-Procesi theorem (cf. [7]), so we get Armendariz-Steinberg's result with an explicit construction.

We turn now to the question of whether $Q(R)$ can be decomposed into a direct product of simple artinian factors. First observe if $R$ is prime then a simple extension of $R$, easily seen to be $Q(R)$, is obtained merely by inverting elements of $C$ (cf. [8]). Conversely, there is

Theorem 5. Let a semiprime $\Omega$-algebra $T$ be essential as a left $R$-module extension of $R$. Then for any inessential prime ideal $P$ of $T, P \cap R$ is an inessential prime ideal in $R$.

Proof. $0 \neq R \cap A \mathrm{nn}_{T} P \subseteq{A \mathrm{nn}_{R}}_{R}(P \cap R)$, so $P \cap R$ is inessential in $R$. To see $P \cap R$ is prime in $R$, let $A, A^{\prime}$ be ideals of $R$ such that $A A^{\prime} \subseteq P$, and pick $q$ arbitrarily in $T$. Since $Q(R)$ is the maximal left essential extension of $R, T \subseteq$ $Q(R)$ as left $R$-modules, so (by Theorem 1 (iii)) there is an essential ideal $E$ of $C$ such that $E q \subseteq R$. Let $B=A_{n n}(P \cap R) . E B A q A^{\prime}=B A E q A^{\prime} \subseteq B A A^{\prime}=0$, so $B A q A^{\prime}=0$ by Theorem 1 (iv). But $B \nsubseteq P$ since $T$ is semiprime, so $A q A^{\prime} \subseteq P$, all $q$ in $T$. Hence $A T A^{\prime} \subseteq P$, implying $A \subseteq R \cap P$ or $A^{\prime} \subseteq R \cap P$. Q.E.D.

Incidentally, it is well known and very easily seen that the module injection $T C$ $Q(R)$ is in fact a ring injection.

Corollary 5. Let $T$ be as in the theorem. If $T$ is prime then $R$ is prime (and thus $Q(R)$ is simple artinian).

Proof. 0 is an inessential ideal of $T$, so 0 is prime in R. Q.E.D.

Call a ring prime-essential if all its primes are essential. Prime-essential semiprime PI-algebras exist, as shown in [9].

Corollary 6. If $R$ is prime-essential then $T$ is prime-essential.

Proof. Immediate from the theorem.

In [9], under the assumption $R$ has zero left singular ideal (not necessarily a PI-algebra), $Q(R)$ is given canonically as the complete direct product of maximal left quotients of prime images and the maximal left quotient of a prime-essential 
ring, the latter being 0 if and only if $\bigcap\{P \mid P$ inessential prime ideal of $R\}=0$. Hence, in view of Theorem 5, we have immediately

Theorem 6. $Q(R)$ is the direct product of simple algebras and a prime-essential algebra. There is a direct summand, simple as an algebra, of $Q(R)$ if and only if $R$ bas an inessential prime ideal. There is an algebra $T \supseteq R$, essential as a left $R$-module and a product of simple algebras, if and only if the intersection of the inessential primes of $R$ is 0 ; in this case we can take $T=Q(R)$.

4. Maximal quotients of semiprime PI-algebras with involution. A semiprime Pl-algebra with involution $(R, *)$ is a semiprime PI-algebra with antiautomorphism $\left(^{*}\right)$ of degree $\leq 2$. Note $(*)$ is an automorphism of degree $\leq 2$ on $C$. Define $\operatorname{cent}(R, *)=\left\{c \in C \mid c^{*}=c\right\}$, and let $\hat{C}=\operatorname{cent}(R, *)$. If $\hat{C}=C$ then $\left(^{*}\right)$ is of first kind on $R$; otherwise $(*)$ is of second kind on $R$.

Theorem 7. If $(R, *)$ is a semiprime PI-algebra with involution then $Q(R)$ bas an involution of the same kind as (*), coinciding with $(*)$ on $R$.

Proof. We use the characterization of $Q(R)$ in Theorem 1. Given $q$ in $Q(R)$, choose an essential ideal $E$ of $C$ such that $E q \subseteq R$. It is easy to show $E^{*}=$ $\left\{c \in C \mid c^{*} \in E\right\}$ is an essential ideal of $C$; define $f: E^{*} \rightarrow R$ by $f(x)=\left(x^{*} q\right)^{*}$ for each $x$ in $E^{*}$. For any $c$ in $C, f(c x)=\left(x^{*} c^{*} q\right)^{*}=\left(c^{*} x^{*} q\right)^{*}=\left(x^{*} q\right)^{*} c=f(x) c=$ $c f(x)$, so $f \in \operatorname{Hom}_{C}\left(E^{*}, R\right)$. Hence there is an element of $Q(R)$, which we shall call $q^{*}$, such that $x q^{*}=f(x)$ for all $x$ in $E^{*}$. A straightforward verification shows $q^{*}$ is independent of the choice of $E$, and $q \rightarrow q^{*}$ is an involution, coinciding with the given involution on $R$. In particular, if $\left(^{*}\right)$ is of the second kind on $R$ then cent $\left(Q(R),{ }^{*}\right) \neq$ cent $Q(R)$, so $\left(^{*}\right)$ is of the second kind on $Q(R)$.

On the other hand, suppose $\left(^{*}\right)$ is of the first kind on $R$. Then, with notation as above, $E^{*}=E$ and $f$ is given by $f(x)=(x q)^{*}$ for each $x$ in $E^{*}$. If $q \in$ cent $Q(R)$ then $x q \in R \cap$ cent $Q(R)=C$, so $f(x)=x q$; therefore $q^{*}=q$ and $\left(^{*}\right)$ is of the first kind on $Q(R)$.

An ideal of $(R, *)$ is an ideal of $R$, stable under $(*)$; an ideal of $(R, *)$ is essential if it intersects nontrivially each nonzero ideal of $(R, *)$.

Lemma 3. (i) If $A$ is an ideal of $(R, *)$ then Ann $A$ is an ideal of $(R, *)$.

(ii) If $J$ is an essential ideal in $(R, *)$ then $J$ is essential in $R$.

(iii) If $J$ is an essential ideal of $R$ then $J^{*}$ is essential in $(R, *)$.

Proof. (i) Let $B=A n n A . B^{*} A=\left(A^{*} B\right)^{*}=(A B)^{*}=0$, so $B^{*} \subseteq A$ nn $A=B$; by symmetry, $B=B^{*}$.

(ii) $(J \cap A n n J)^{2} \subseteq J$ Ann $J=0$, implying $J \cap A n n J=0$. But Ann $J$ is an ideal of $(R, *)$, so Ann $J=0$, implying $J$ is essential in $R$.

(iii) $J^{*}$ is clearly essential in $R$, so $J J^{*}$ is essential in $R$; thus $J J^{*}$ is certainly essential in $(R, *)$. Q.E.D. 
Theorem 8. Let $\hat{C}=\operatorname{cent}(R, *) .(Q(R), *)$ can be characterized as follows:

(i) There is an injection $(R, *) \rightarrow(Q(R), *)$ sending $\hat{C}$ into cent $\left(Q(R), *^{*}\right)$.

(ii) For any essential ideal $E$ of $\hat{C}$ and for any $f$ in $\operatorname{Hom}_{\hat{C}}(E, R)$, there exists $q$ in $Q(R)$ such that $x q=f(x)$, all $x$ in $E$.

(iii) Given $q$ in $Q(R)$, one can find an essential ideal $E$ of $\hat{C}$ sucb that $E q \subseteq R$.

(iv) $q=0$ if and only if there exists an essential ideal $E$ of $\hat{C}$ sucb that $E q=0$.

Proof. This is straightforward from Theorems 1 and 7, when it is noted that $\hat{C}=$ cent $(C, *)$; hence any essential ideal of $\hat{C}$ is an essential ideal of $C$ by Lemma 3, and if $E$ is an essential ideal of $C$ then $E E^{*}$ is an essential ideal of $\hat{C}$.

Conversely, we wish to show that for any algebra $\left(Q,{ }^{*}\right)$ satisfying $(i)^{\prime}-(i v)^{\prime}$, $Q$ is the maximal quotient algebra of $R$. To see this, we shall show $Q$ satisfies properties (i)-(iv) of Theorem 1. Observe that, by Lemma 3, any essential ideal in $\hat{C}$ is essential in $C$.

Hence (iii) and (iv) are immediate. To obtain (i), we need only show $C \subseteq$ cent $Q$. Indeed, given $c \in C$ and $q$ in $Q$, choose an essential ideal $E$ of $\hat{C}$ such that $E q \subseteq R$. Then $E(c q-q c)=0$, so $c q-q c=0$ for all $q$ in $Q$, implying $c \in$ cent $Q$.

Finally we need to prove (ii). Suppose $E$ is an essential ideal of $C$ and $f \in \operatorname{Hom}_{C}(E, R)$. Then $E^{*} E$ is essential in $\hat{C}$ and $f$ restricts to a $\hat{C}$-homomorphism from $E^{*} E$ to $R$; hence there is $q$ in $Q$ such that $f(x)=x q$, all $x$ in $E^{*} E$. Thus, for all $x$ in $E, E^{*}(f(x)-x q)=0$, implying $f(x)-x q=0$, all $x$ in $E$ by (iv). Q.E.D.

\section{REFERENCES}

1. S. A. Amitsur, On rings of quotients, Istituto Nazionale di Alta Matematica, Symposia Matematica, vol. VIII, 1972.

2. E. Armendariz and S. Steinberg, Regular self-injective rings with a polynomial identity, Trans. Amer. Math. Soc. 190 (1974), 417-425.

3. J. W. Fisher, Structure of semiprime P.I. rings. I, Proc. Amer. Math. Soc. 39 (1973), 465-467.

4. E. Formanek, Central polynomials for matrix rings, J. Algebra 23 (1972), 129-132. MR $46 \# 1833$.

5. R. E. Johnson, Quotient rings of rings with zero singular ideal, Pacific J. Math. 11 (1961), 1385-1392. MR 26 \#1331.

6. W. S. Martindale III, On semiprime P.I. rings, Proc. Amer. Math. Soc. 40 (1973), 365-369.

7. C. Procesi, On a theorem of M. Artin, J. Algebra 22 (1972), 309-315. MR 46 $\# 1825$.

8. L. H. Rowen, Some results on the center of a ring with polynomial identity, Bull. Amer. Math. Soc. 79 (1973), 219-223.

9. - A subdirect decomposition of semiprime rings and its application to maximal quotient rings, Proc. Amer. Math. Soc. (to appear). 